\title{
A Method of Selecting the Block Size of BMM for Estimating Extreme Loads in Engineering Vehicles
}

\author{
Jixin Wang, ${ }^{1}$ Shuang You, ${ }^{1}$ Yuqian Wu, ${ }^{1}$ Yingshuang Zhang, and Shibo Bin ${ }^{2}$ \\ ${ }^{1}$ School of Mechanical Science and Engineering, Jilin University, Changchun 130025, China \\ ${ }^{2}$ LiuGong Machinery Co., Ltd., Liuzhou 545000, China \\ Correspondence should be addressed to Jixin Wang; jxwang@jlu.edu.cn
}

Received 30 July 2016; Accepted 22 September 2016

Academic Editor: Eric Feulvarch

Copyright (C) 2016 Jixin Wang et al. This is an open access article distributed under the Creative Commons Attribution License, which permits unrestricted use, distribution, and reproduction in any medium, provided the original work is properly cited.

\begin{abstract}
Extreme loads have a significant effect on the fatigue damage of components. The block maximum method (BMM) is widely used to estimate extreme values in various fields. Selecting a reasonable block size for BMM is crucial to ensure that proper extreme values are extracted to get extreme sample to estimate extreme values. Aiming at this issue, this study proposed a comprehensive evaluation approach based on multiple-criteria decision making (MCDM) method to select a proper block size. A wheel loader with six sections in one operating cycle was illustrated as an example. First, spading sections of each operating cycle were extracted and connected as extreme loads often occur at that section. Then extreme sample was obtained by BMM for fitting the generalized extreme value (GEV) distribution. Kolmogorov-Smirnov (K-S) test, Pearson's Chi-Square $\left(\chi^{2}\right)$ test, and average deviation in Probability Distribution Function (PDF) are selected as the fitting test. The comprehensive weights are calculated by the maximum entropy principle. Finally, the optimal block size corresponding to the minimum comprehensive evaluation indicator is obtained and the result exhibited a good fitting effect. The proposed method can also be flexibly used in various situations to select a block size.
\end{abstract}

\section{Introduction}

Load analysis should be considered during vehicle design [1]. First, a measured load can be regarded as a sequence of turning points formed by the local maxima and minima, which determine the amplitude of load cycles. But the measured load represents only a very limited part of the design life and necessarily should be extrapolated in the time domain if our main goal is to perform fatigue tests or FEM fatigue analysis. The main issue in load analysis is how to determine the extreme cycles [2], which contribute to fatigue damage significantly. Second, the components of transmission cannot contain any growing cracks when subjected to millions of cycles in a short period of time. The extreme loads are therefore the most important load characteristics that determine a fatigue limit. Third, the components of engineering vehicles may bear extreme loads caused by misuse and rare events and will be severely damaged, and even sudden failures may occur. As extreme loads appear once or a few times in the design life, they should be estimated from the measured load. Therefore, when the fatigue limit, life prediction, and sudden failures are considered for designing mechanical components in the time domain or amplitude domain, extreme loads should be estimated accurately to achieve the correct balance between safety design and overdesign [3].

Extreme value theory (EVT), based on asymptotic theory, provides a model that can be extrapolated to deduce the probability of certain serious extreme events, which have not happened or have not been recorded yet. EVT can be applied to estimate extreme values in various fields, such as wind engineering, strength of materials, and fatigue strength [46]. To fit the extreme distribution, extreme sample should be extracted accurately from the measured load. The most commonly used methods are BMM, Peaks over Threshold (POT), and the method of independent storms (MIS). POT method [7] is applicable for selecting loads above a threshold, but the crucial problem is how to determine the threshold properly [8-10]. Although there are many researches about the threshold, a stable and effective method has not been established. MIS [11] selects maximum loads of each independent storm and has a lower sensitivity than POT in threshold 
determination, but it requires a longer measured load for a few loads which remained after using MIS.

BMM has been widely used to estimate extreme values, especially in dealing with seasonal data. Selecting a proper block size for BMM is crucial [12], the methods of selecting the block size vary from different fields, such as wind speeds [13], flood [14], rainfall [15], and other fields [16-18]. In the fields mentioned above, such as wind speeds, we assume that $Y_{1}, Y_{2}, \ldots, Y_{n}$ are the daily observations. The block size is set as a year and $Z_{i}$ is the annual maximum because the wind speeds undergo seasonal variations in a year. Given further research on extreme loads in engineering vehicles, how to select a block size properly is still a problem. This study took a wheel loader as an example. Its fixed V-6 operation pattern was used in the experimental measurement of service loadings $[19,20]$, that is, no load forward, spading, full load backward, full load forward, unloading, and load backward, as exemplified in Figure 1(a). The actual measured load time history at a drive flange under the V-6 operating scheme was shown in Figure 1(b); the change among different sections is similar to the seasonal characteristics of wind speeds. In wind speeds, one year is commonly used as a block size, while in engineering vehicles one operating cycle may reasonably be set as a block size. Then the maximum loads of each operating cycle were extracted to fit GEV. However, the fitting effect of GEV shown in the latter part of the paper was not ideal.

To extract more extreme loads and model a reliable extreme distribution by restricting the fitting error of GEV, spading sections of each operating cycle were extracted and connected. Then a comprehensive evaluation approach based on MCDM was proposed to select a proper block size for BMM. The block size is determined by comparing the comprehensive fitting errors with less subjectivity and prejudice. The load analysis in this paper was realized through WAFO and EVIM [21, 22], and it can flexibly be used in various situations to select a block size.

\section{GEV for Extreme Loads Analysis}

2.1. Extreme Distribution. If the constant numbers $\left\{a_{n}>0\right\}$ and $\left\{b_{n}\right\}$ exist and satisfy the equation

$$
\begin{aligned}
\operatorname{Pr}\left\{\frac{M_{n}-b_{n}}{a_{n}} \leq x\right\} & =\operatorname{Pr}\left\{M_{n} \leq a_{n} x+b_{n}\right\} \\
& =F^{n}\left(a_{n} x+b_{n}\right) \longrightarrow H(x),
\end{aligned}
$$

$x \in R$,

where $H(x)$ is a nondegraded function, then $H(x)$ must be one of the three forms:

$$
\begin{aligned}
& \text { Gumbel: } H_{1}(x)=\exp \left(-\exp ^{-(x-\mu) / \sigma}\right), \\
& -\infty<x<+\infty, \\
& \text { Fréchet: } H_{2}(x, \alpha)= \begin{cases}0, & x \leq \mu, \\
\exp \left\{-\left(\frac{x-\mu}{\sigma}\right)^{-\alpha}\right\}, & x>\mu,\end{cases} \\
& \text { Weibull: } H_{3}(x, \alpha)= \begin{cases}\exp \left\{-\left(\frac{x-\mu}{\sigma}\right)^{\alpha}\right\}, & x \leq \mu, \\
1, & x>\mu .\end{cases}
\end{aligned}
$$

As for Fréchet and Weibull distributions $(\alpha>0)$, the probability density functions of them are illustrated in Figure 2.

Only one of the three forms is practically selected to undertake a desirable fitting from the sample load; its distribution parameter is then estimated. However, it is difficult to decide which of the three functions has the best fitting effect. Besides, the selection is performed based on earlier experience. Thus, arbitration plays a high-weighted role during the process, and a mistaken selection of the distribution form leads to disastrous estimated results. Therefore, integrating the three forms of distribution into a comprehensive one, namely, GEV, is preferable:

$$
H(x ; \mu, \sigma, \xi)=\exp \left[-\left(1+\xi \frac{x-\mu}{\sigma}\right)^{-1 / \xi}\right],
$$

where $1+\xi(x-\mu) / \sigma>0, \xi, \sigma, \mu$, respectively, represent the shape, scale, and position parameters, and all of them satisfy the demand that $\xi \in R, \sigma>0, \mu \in R$. The tail form of GEV is determined solely by $\xi$; a higher value of $\xi$ indicates a thicker tail; hence, $\xi$ is defined as extreme value index. The relationship of GEV and the three extreme value distributions depends on the value of $\xi$. If $\xi=0$, GEV refers to a Gumbel distribution; if $\xi>0$, GEV refers to a Fréchet distribution; and if $\xi<0$, GEV refers to a Weibull distribution.

2.2. Extreme Sample Selected by BMM. The extraction of extreme values is significant when GEV is applied to fit the tail of the data. Suppose that the continuous sampling data $Y_{1}, Y_{2}, \ldots, Y_{n}$ are divided into $k$ blocks with the interval length $l$. The maximum loads $M_{l_{1}}, M_{l_{2}}, \ldots, M_{l_{k}}$ of each block are extracted to constitute extreme sample, as depicted in Figure 3. And $M_{l_{1}}, M_{l_{2}}, \ldots, M_{l_{k}}$ can be viewed as independent identically distributed observations from GEV. However, if a selected block size is unreasonable, a large scale of extreme loads will be wasted. The fitting will be inaccurate because a block size that is too small may lead to biased estimation, whereas a one that is too large may result in few extracted extreme values and subsequently a large variance. Thus, to fully extract extreme values to constitute sample loads for fitting GEV, the block size must be exactly selected.

Practically, $Y_{i}$ is sample data obtained during some process or some period of time, such as average temperature per day or average wind velocity per $10 \mathrm{~min} ; M_{n}$ is the maximum value corresponding to the process or time. The period of time for perspicuously seasonal data is often a year; $M_{n}$ represents the annual maximum value. Though under different working conditions, a loader's average working time for each operating cycle is $36 \mathrm{~s}$; the components of a loader work under periodically loading conditions. One or more extreme loads may occur in each operating cycle. In a short period of time, numerous extreme loads will have an impact on the components. Thus, it is imperative to find an efficient method that differs from the traditional method applied in other fields to select block size and estimate extreme loads in engineering vehicles.

2.3. Parameter Estimate Using MLE. Maximum likelihood estimation (MLE) method is a kind of point estimation and 


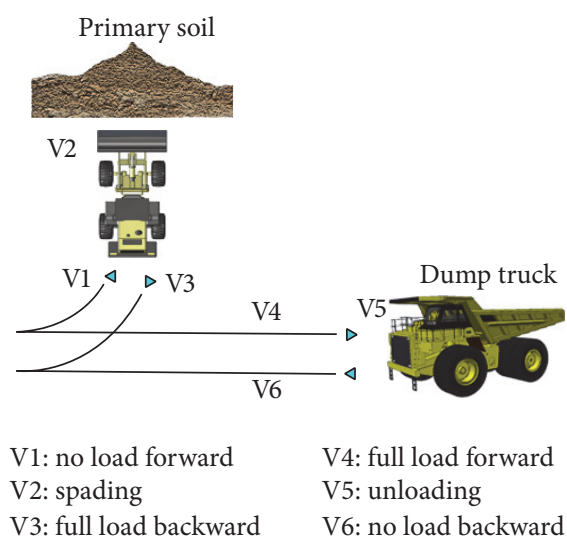

(a)

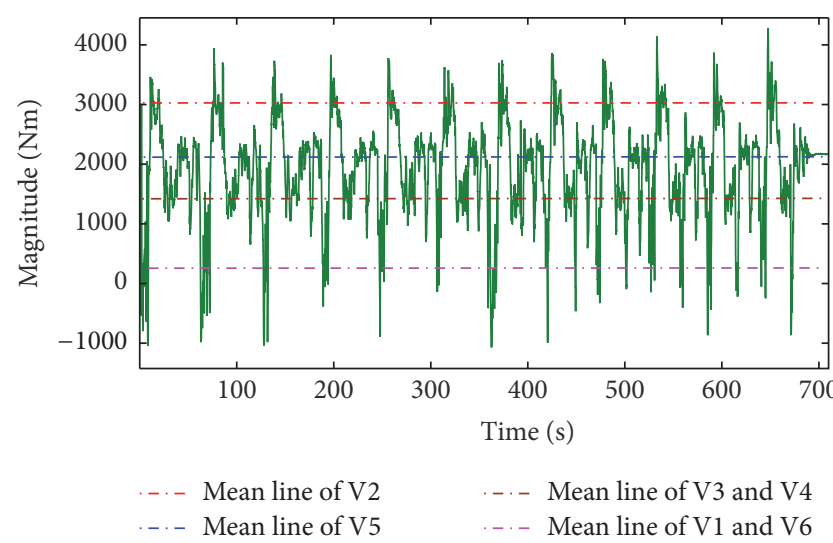

(b)

FIgURE 1: (a) V-6 operating scheme. (b) Actual load history measured at the drive flange of a wheel loader.

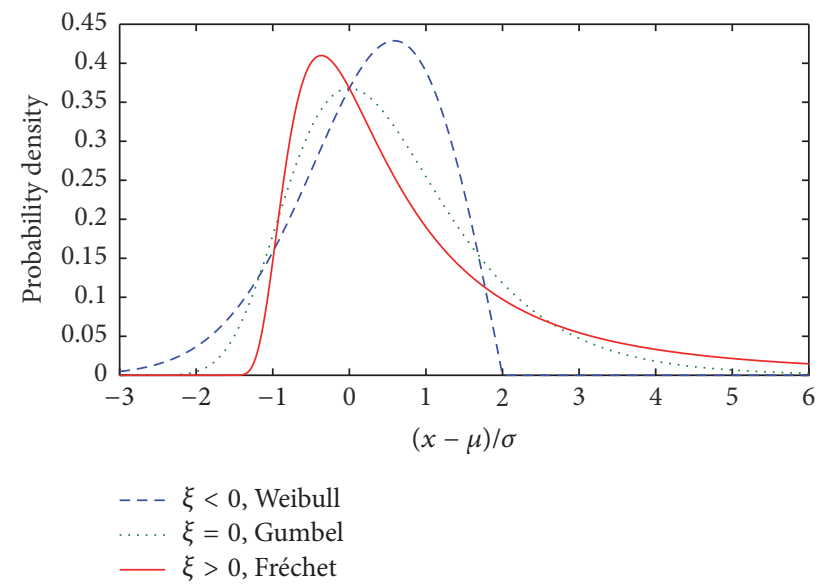

FIGURE 2: Probability density function of Gumbel, Fréchet, and Weibull.

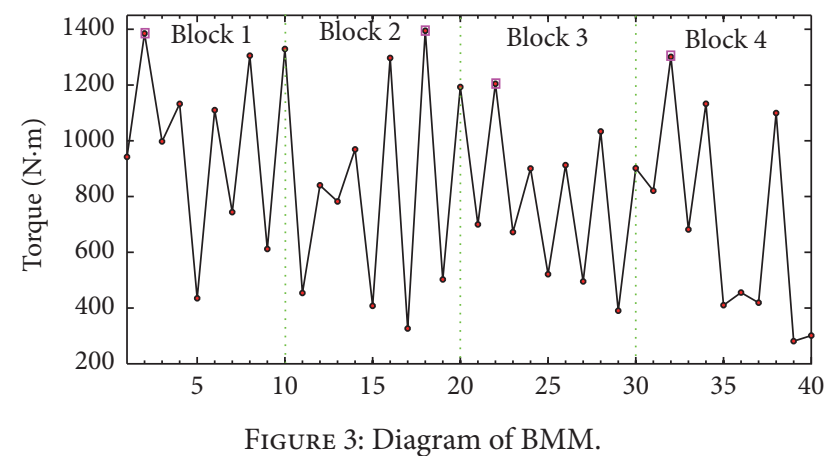

the most popular method applied in extreme loads estimation. Its superiority lies in asymptotic normality, congruency, invariance, and other attributes when the distribution is known. Hosking [23] provided the arithmetic procedure for parameter estimation with maximum likelihood method.

$X_{1}, X_{2}, \ldots, X_{n}$ are independent and identically distributed, whose log-likelihood function is as follows.
When $\xi=0$,

$$
\begin{aligned}
L(\sigma, \mu)= & -k \log \sigma \\
& -\sum_{i=1}^{k}\left(\frac{x_{i}-\mu}{\sigma}\right)-\sum_{i=1}^{k} \exp \left(-\frac{x_{i}-\mu}{\sigma}\right) .
\end{aligned}
$$

When $\xi \neq 0$ and satisfies $1+\xi\left(\left(x_{i}-\mu\right) / \sigma\right)>0$,

$$
\begin{aligned}
L(\xi, \sigma, \mu)= & -k \log \sigma \\
& -\left(1+\frac{1}{\xi}\right) \sum_{i=1}^{k} \log \left[1+\xi\left(\frac{x_{i}-\mu}{\sigma}\right)\right] \\
& -\sum_{i=1}^{k}\left[1+\xi\left(\frac{x_{i}-\mu}{\sigma}\right)\right]^{1 / \xi},
\end{aligned}
$$

where $k$ is block numbers in the BMM model; $\xi, \sigma, \mu$ can be solved through the two equations above.

After a thorough study on the application of maximum likelihood estimation in GEV, Smith discovered that when $\xi \in$ $(-0.5,+\infty)$, the parameter in MLE can be obtained and it is holomorphic; when $\xi \in(-1,-0.5)$, the parameter in MLE can also be obtained but has no generally asymptotic property; when $\xi \in(-\infty,-1)$ the parameter in MLE cannot be obtained any more. The second and third conditions seldom occur when applying GEV for parameter estimation. Therefore, the theoretical defect of MLE poses little harm to its practical application.

2.4. Test of Goodness-of-Fit. The test of goodness-of-fit is primarily used to evaluate the selected block size. The graphic and calculation methods are available for the test. The quantile-quantile (Q-Q) plot and probability-probability (P$\mathrm{P})$ plot are graphic methods for comparing two probability distributions or two data sets. If they are similar and close, the points in the Q-Q and P-P plots will approximately lie on a line. There are many kinds of calculation methods, the most popular methods are the Kolmogorov-Smirnov (K-S) test, Pearson's Chi-Square $\left(\chi^{2}\right)$ test, average deviation in PDF, 


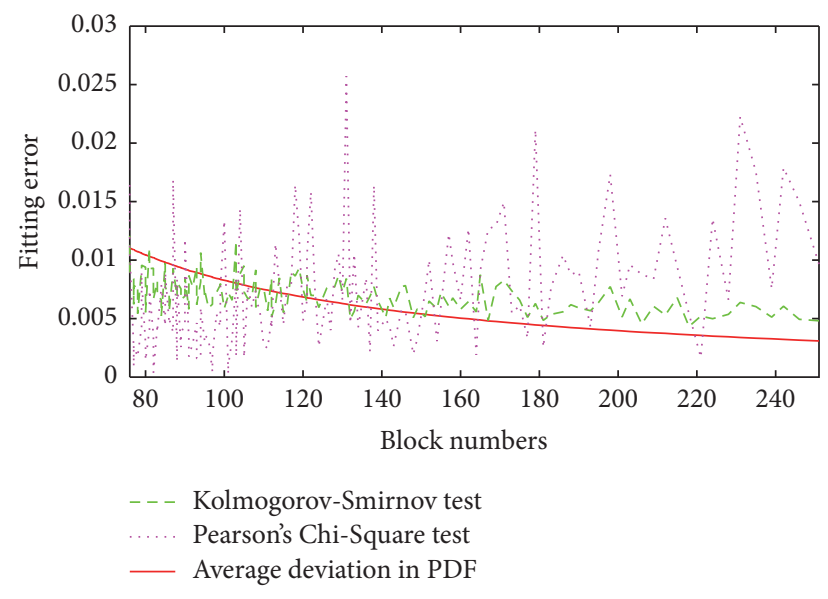

FIGURE 4: Standardized fitting error of test methods.

root mean square deviation, and correlation coefficient test $\left(R^{2}\right)[24,25]$. This study selected three tests to describe the fitting effect of GEV; the details are described as follows:

$$
\begin{aligned}
K_{\max } & =\max _{1 \leq j \leq n-1}\left\{\left|F\left(x_{i}\right)-G\left(x_{i}\right)\right|,\left|F\left(x_{i+1}\right)-G\left(x_{i}\right)\right|\right\}, \\
\chi^{2} & =\sum_{i=1}^{n} \frac{\left(O_{i}-E_{i}\right)^{2}}{E_{i}} \\
\delta_{\mathrm{pdf}} & =\frac{1}{n} \sum_{i=1}^{n}\left|F\left(x_{i}\right)-g\left(x_{i}\right)\right| .
\end{aligned}
$$

The K-S test quantifies the distance between the hypothesized cumulative distribution function (CDF) and PDF. The smaller $K_{\max }$, the better the goodness-of-fit. $\chi^{2}$ test measures the distance between the observed data and the normal distribution with mean $0 ; O_{i}$ is the observed count, and $E_{i}$ is the expected count. The hypothesized distribution represents the observation data better when $\chi^{2}$ is smaller [26]. For the average deviation in PDF, smaller $\delta_{\text {pdf }}$ means that a hypothesized distribution can represent the observation data better.

\section{Comprehensive Evaluation Approach Based on MCDM}

The graphic method of the test of goodness-of-fit is too subjective, whereas each of the tests in calculation method can only test the goodness-of-fit in one certain aspect. Given that different researchers adopt different tests, the determined block size may be various. Figure 4 shows that when adopting different block sizes in a sample load for fitting GEV, K-S test, $\chi^{2}$ test, and average deviation demonstrate different rules of variation. When test methods are used to determine the block size, respectively, the results vary greatly. Therefore, it is crucial to find a method that comprehensively considers different fitting test results to select the block size properly.

The comprehensive evaluation approach based on MCDM [27-29] can integrate different tests into a comprehensive one that considers different results from the various tests and makes the selection of block size more scientific and objective. The key point of the comprehensive evaluation approach is to determine the weight of each test. The entropy value method (EVM) determines the weight of each test based on its respective effect on block size and is a relatively objective weight-determining method. According to the maximum entropy principle and the combination of the three tests, an exact method of determining block size was proposed in this study. Its procedure is shown in Figure 5.

3.1. Calculating the Weight of the Tests. Entropy was first proposed by German scientist R. Clausius in 1846. Claude Elwood Shannon introduced this concept to the theory of information, where it was used to measure the degree of nondeterminacy [30]. Since its proposition, the concept of entropy has been introduced to many fields such as probability theory, bioscience, and engineering.

In the present study, the selected block sizes in each test are different. Aiming at the content, $U_{1}, U_{2}, \ldots, U_{m}$ are the candidate block sizes and $T_{1}, T_{2}, \ldots, T_{n}$ are the tests. The error of fitting from applying $T_{j}$ to $U_{i}$ is $x_{i j}$, and the evaluation matrix $X$ is shown as follows:

$$
\begin{array}{cccccc} 
& T_{1} & \cdots & T_{j} & \cdots & T_{n} \\
& w_{1} & & w_{j} & & w_{n} \\
U_{1} & x_{11} & \cdots & x_{1 j} & \cdots & x_{1 n} \\
\vdots & \vdots & \ddots & & & \vdots \\
U_{i} & x_{i 1} & & x_{i j} & & x_{i n} \\
\vdots & \vdots & & \vdots & \ddots & \vdots \\
U_{m} & x_{m 1} & \cdots & x_{m j} & \cdots & x_{m n}
\end{array}
$$

where $w_{1}, w_{2}, \ldots, w_{n}$ are unknown parameters; those values need be calculated by EVM.

For $T_{j}$, a higher varying magnitude of $x_{i j}$ from fitting with candidate $U_{i}$ indicates a larger entropy. This larger entropy implies that $T_{j}$ has a larger influence on $U_{i}$. In addition, a lower varying magnitude of $x_{i j}$ implies that $T_{j}$ has a smaller influence on $U_{i}$. If for certain $T_{j}$, its error of fitting on every block size is congruent, then $T_{j}$ has nothing to do with the ultimate decision and its weight $w_{j}$ is 0 .

The evaluation matrix needs to be standardized. The normalization method reserves the degree of effect of the tests on evaluation objectives and is adopted in this study.

$$
\widehat{x}_{i j}=\frac{x_{i j}}{\sum_{i=1}^{m} x_{i j}}, \quad(1 \leq i \leq m, 1 \leq j \leq n),
$$

where $\hat{x}_{i j}$ represents the standardized $x_{i j}$; the column represents the evaluation objectives; thus, $\sum_{i=1}^{m} \hat{x}_{i j}=1$.

The entropy $E_{j}$ from test $T_{j}$ can be calculated as follows:

$$
E_{j}=-\frac{1}{\ln m} \sum_{i=1}^{m} \widehat{x}_{i j} \ln \widehat{x}_{i j}
$$

where $0<E_{j}<1$. 


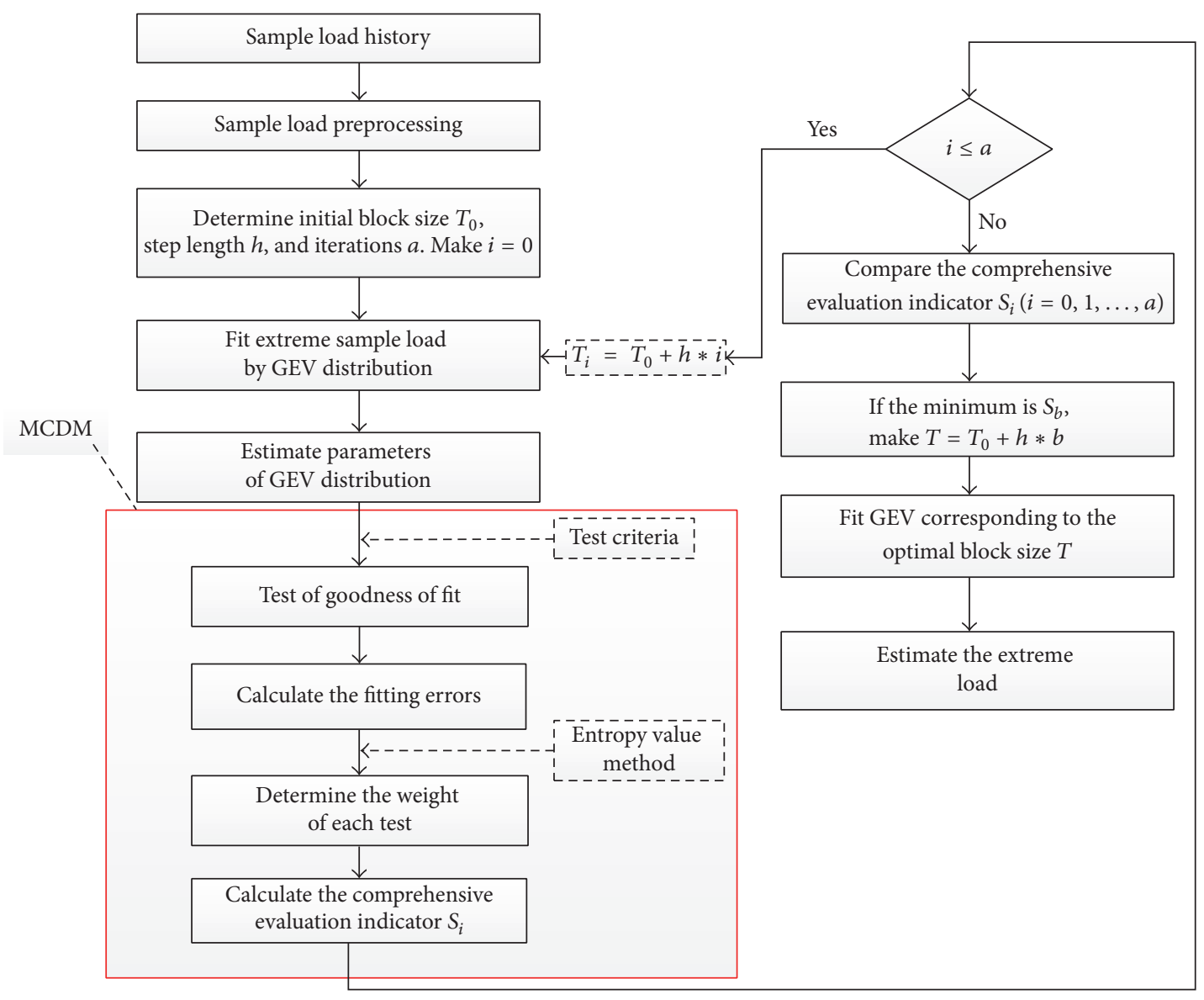

FIGURE 5: Flow chart for determining block size based on MCDM.

The entropy weight of each test can be calculated as follows:

$$
w_{j}=\frac{1+(1 / \ln m) \sum_{i=1}^{m} \widehat{x}_{i j} \ln \widehat{x}_{i j}}{\sum_{k=1}^{m}\left(1+(1 / \ln m) \sum_{i=1}^{m} \widehat{x}_{i j} \ln \widehat{x}_{i j}\right)} .
$$

The weight vector is $w=\left(w_{1}, w_{2}, \ldots, w_{n}\right)$. The comprehensive evaluation indicator that integrates different tests can be calculated as follows:

$$
s=\widehat{x}_{i j} \times w .
$$

When fitting for GEV is conducted, the comprehensive error calculated by (11) should be small enough.

\section{Case Study}

In the field of engineering vehicles, extreme loads to which the components are subjected should be estimated. Extreme loads usually occur once or more during one operating cycle because of the tough working conditions. Traditionally, when the BMM is adopted, the selection of block size is based on the engineering practice of technicians, and the graphic method is applied to subjectively evaluate the fitting effect, which depends heavily on the experience of data processing. A wheel loader was taken as an illustrative example to verify the effectiveness and practicability of the proposed method.
4.1. Data Analysis. A wheel loader's fixed V-6 operation pattern for loading and unloading bulk material was used in the experimental measurement under spading primary soil. The actual measured load history of an axle shaft measured at 75 operating cycles was obtained. After denoising, smoothing, and other pretreatments conducted on the data, selecting one operating cycle as a block size may be reasonable because of the section working characteristics of a wheel loader, which are analogous to the seasonal characteristics of wind speeds. However, the fitting effect of GEV was not ideal, as shown in Figure 6. The extreme information was wasted when the block size is set as one operating cycle because of the increased probability of the appearing extreme loads. Therefore, the loads of six sections in each operating cycle were individually extracted, and then the loads of the same section were combined to form a new load history, as shown in Figure 7. Given that the spading section was more serious than any other sections, the connected spading section was extracted.

Because the data of each spading section enjoy the same load characteristic, the connected data were independent and identically distributed, which adhere to the underlying theoretical basis for the GEV indicating that the parent population is independent and identically distributed.

In this study, a new load time history was obtained by combining the data of the spading section of each operating 

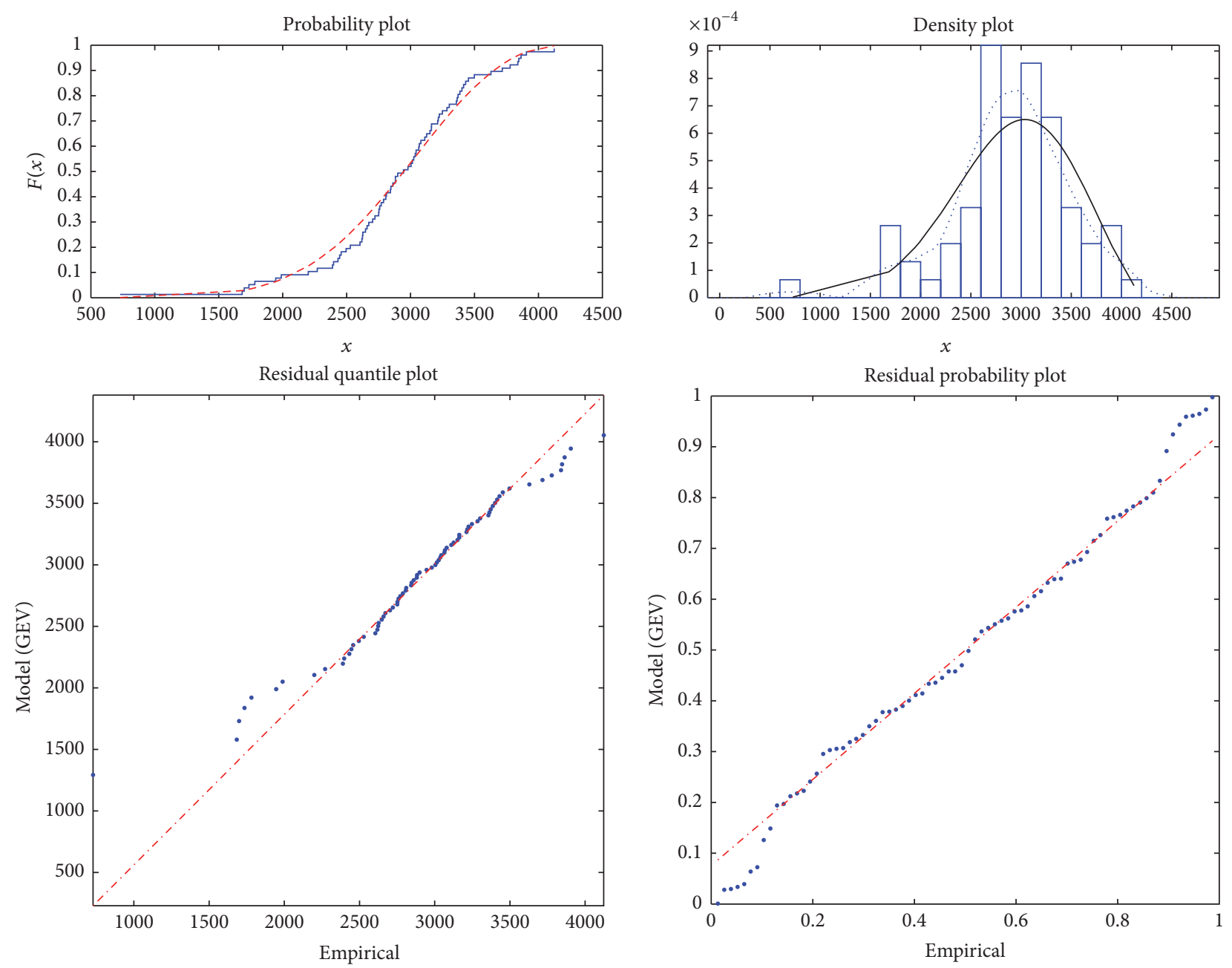

Fit method: ML; fit $p$ value: 0.86

FIgURE 6: GEV fitting inspection under block size $=1000$.

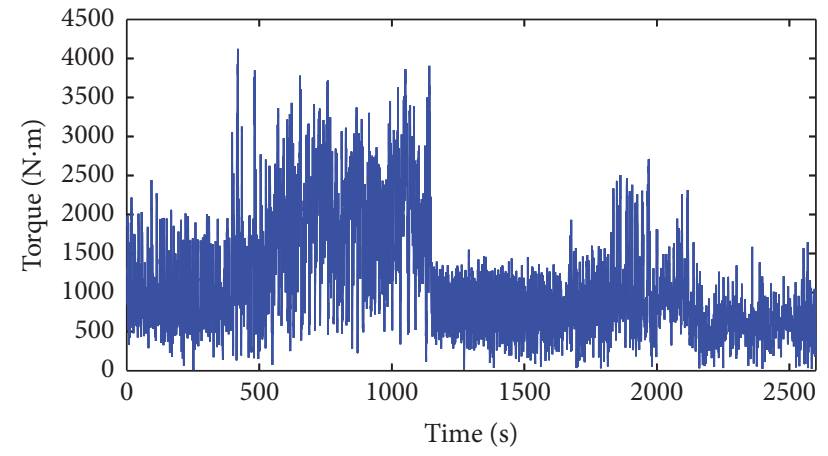

Figure 7: New load history after extracting and combining the data from each section.

cycle, and the block size was selected using the comprehensive evaluation approach to obtain more extreme loads. The working time for every spading section is $10 \mathrm{~s}$. A hundred data points are gathered per second, and the total time is $750 \mathrm{~s}$. Therefore, 75001 data points were gathered, as shown in Figure 8.

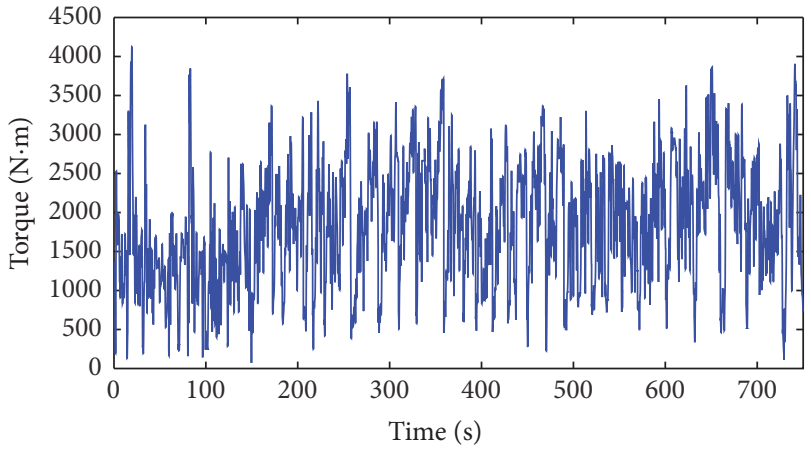

Figure 8: Load data of the combined spading sections.

4.2. Determination of the Comprehensive Evaluation Indicator. Based on the proposed method in this study, a third of the number of data during a spading section was selected as the initial block size and the number of data during a spading section was selected as the terminal condition. The region of the block size was $[300,1000] ; 5$ data points were set 


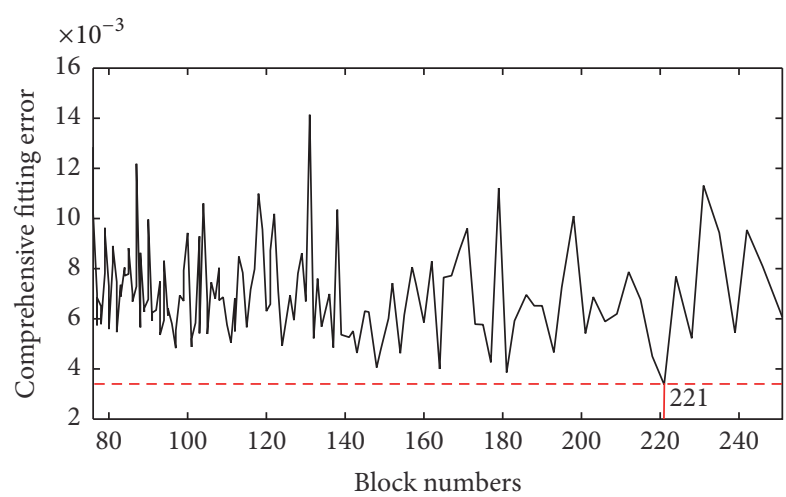

FIgURE 9: Comprehensive error of fitting GEV.

as step length. The number of data per block was therefore determined and subsequently the number of blocks. The maximum loads were then extracted to constitute extreme sample to fit GEV. The maximum likelihood function was selected for parameter estimation, and K-S test, $\chi^{2}$ test, and average deviation in PDF were selected for the test of goodness-of-fit. Thus, based on MCDM, a $141 \times 3$ evaluation matrix was constructed. The normalization method was adopted in this study to standardize the estimation matrix because of the different dimensions for the different tests. The standardized evaluation matrix was shown in Table 1.

EVM was adopted to resolve the entropies of the tests, and the entropy vector was resolved. The weight coefficient vector was obtained through (10), and the comprehensive evaluation indicator, that is, the comprehensive fitting error, was calculated through (11).

\subsection{Results and Discussions}

4.3.1. Selecting the Block Size. From Table 1, it can be concluded that when the block number was 218, which corresponded to a block size of 345 , the K-S test reached its nadir. When the block number was 101, which corresponded to a block size of $740, \chi^{2}$ test reached its nadir. When block number was 251, which corresponded to a block size of 300 , the average deviation reached its nadir. Figure 9 showed that when the block number was 221 the comprehensive fitting error reached its nadir and that the block size was 340. As the comprehensive test took all the three tests of K-S, $\chi^{2}$, and average deviation, it can conduct a minimum comprehensive fitting error.

4.3.2. Fitting GEV. Once the block size was selected, the maximum loads of each block were extracted to constitute extreme sample to fit GEV under a confidence region of $95 \%$. The result of fitting GEV was evaluated by adopting the QQ plot, graph of distribution function, graph of probability density function, residue quantile plot, and graph of residue probability density function, as shown in Figures 10 and 11. Compared to the GEV fitted by the extreme sample extracted from each operating cycle, it exhibited a better fitting result, which was compatible with the analytical demand from the BMM. Meanwhile, $p$ value of the first condition was 0.86 and
TABLE 1: Candidate block numbers with standardized matrix.

\begin{tabular}{|c|c|c|c|}
\hline Block numbers & $\begin{array}{c}\text { Mean } \\
\text { deviation }\end{array}$ & K-S & $\chi^{2}$ \\
\hline 251 & 0.0031 & 0.0048 & 0.0097 \\
\hline 246 & 0.0032 & 0.0049 & 0.0150 \\
\hline 242 & 0.0032 & 0.0061 & 0.0179 \\
\hline 239 & 0.0033 & 0.0051 & 0.0076 \\
\hline 235 & 0.0033 & 0.0060 & 0.0175 \\
\hline 231 & 0.0034 & 0.0064 & 0.0223 \\
\hline 228 & 0.0034 & 0.0054 & 0.0066 \\
\hline 224 & 0.0035 & 0.0050 & 0.0135 \\
\hline 221 & 0.0036 & 0.0052 & 0.0017 \\
\hline 218 & 0.0036 & 0.0043 & 0.0054 \\
\hline 215 & 0.0037 & 0.0068 & 0.0094 \\
\hline 212 & 0.0037 & 0.0053 & 0.0136 \\
\hline$\vdots$ & $\vdots$ & $\vdots$ & $\vdots$ \\
\hline 108 & 0.0076 & 0.0092 & 0.0074 \\
\hline 107 & 0.0077 & 0.0069 & 0.0060 \\
\hline 106 & 0.0078 & 0.0067 & 0.0078 \\
\hline 105 & 0.0079 & 0.0073 & 0.0017 \\
\hline 105 & 0.0079 & 0.0095 & 0.0032 \\
\hline 104 & 0.0079 & 0.0088 & 0.0144 \\
\hline 103 & 0.0080 & 0.0071 & 0.0018 \\
\hline 103 & 0.0080 & 0.0116 & 0.0084 \\
\hline 102 & 0.0081 & 0.0066 & 0.0033 \\
\hline 101 & 0.0082 & 0.0072 & 0.0010 \\
\hline 101 & 0.0082 & 0.0070 & 0.0003 \\
\hline$\vdots$ & $\vdots$ & $\vdots$ & $\vdots$ \\
\hline
\end{tabular}

that of the second condition was 0.92. Given that a higher value of $p$ value indicated a more likely GEV distribution, the method was proven to be successful in extracting sufficient extreme values from the measured load and displayed significant engineering practicality for estimating extreme loads.

\section{Conclusions}

When estimating extreme loads in engineering vehicles, block size are sensitive to parameter estimation and the selection of the extreme loads by BMM; thus, selecting a proper block size is essential. Once the BMM is adopted, performing block partition based on engineering practice has the testability and nondeterminacy. To model a reliable extreme distribution by decreasing the fitting error of GEV and evaluate the selected block size, this study proposed a comprehensive evaluation approach based on MCDM to divide blocks automatically and analyze fitting results quantitatively.

The measured load time history of a wheel loader was used as an example to verify the effectiveness and practicability of the proposed method. The block size selected by the proposed method was tested with the graphic method. The results showed that the fitting effect of GEV was desirable, and 


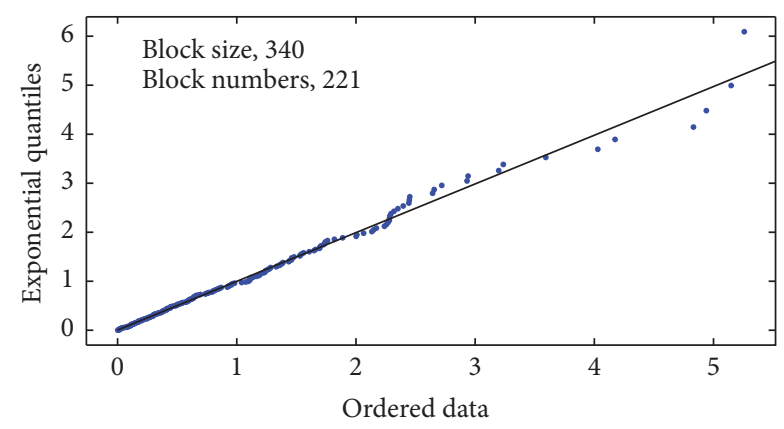

(a)

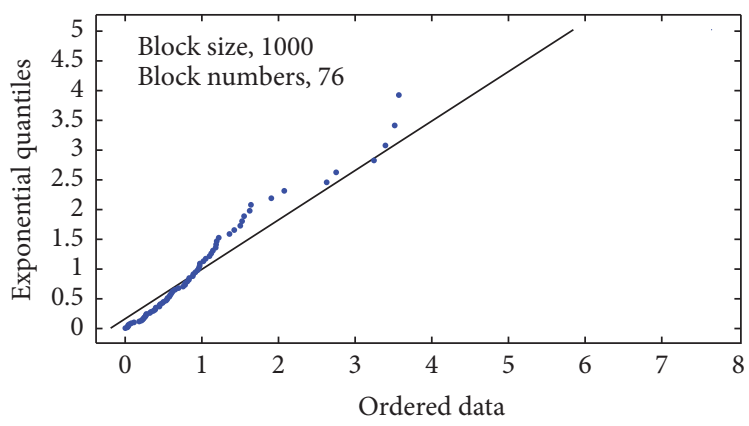

(b)

FIGURE 10: Q-Q plot: (a) block size $=340$; (b) block size $=1000$.
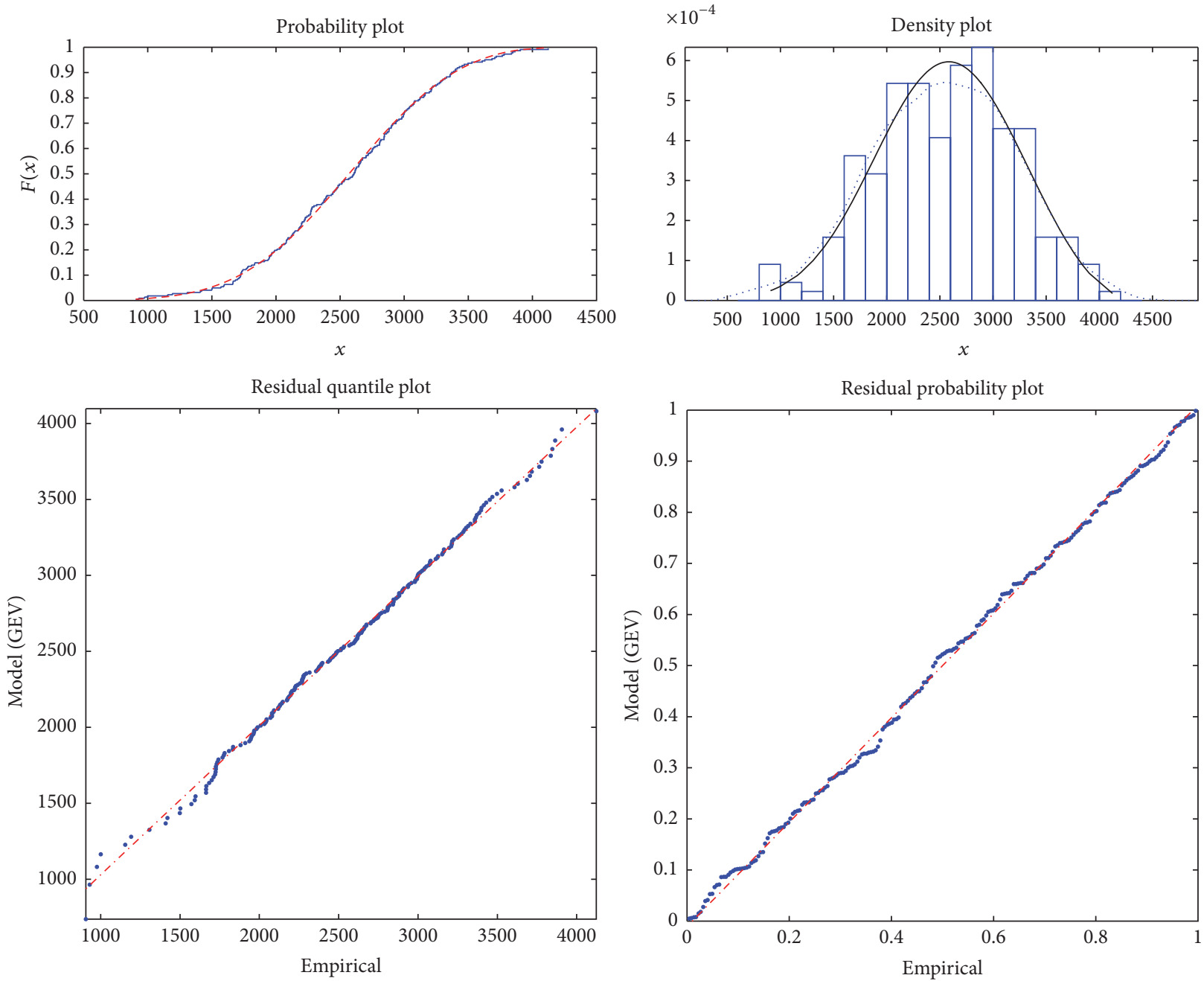

Fit method: ML; fit $p$ value: 0.92

FIGURE 11: GEV fitting inspection under block size $=340$.

then a satisfactory block size was determined. Compared with the GEV fitted by the extreme sample extracted from each operating cycle, it exhibited a better fitting result. The load analysis in this paper decreases the subjectivity and prejudice and can be flexibly used in various situations to select a block size. 


\section{Competing Interests}

The authors declare that they have no competing interests.

\section{Acknowledgments}

This work was supported by National Natural Science Foundation of China (Grant no. 51375202).

\section{References}

[1] P. Johannesson and M. Speckert, Guide to Load Analysis for Durability in Vehicle Engineering, John Wiley \& Sons, New York, NY, USA, 2013.

[2] P. Johannesson, "Extrapolation of load histories and spectra," Fatigue \& Fracture of Engineering Materials \& Structures, vol. 29 , no. 3, pp. 209-217, 2006.

[3] P. J. Moriarty, W. E. Holley, and C. P. Butterfield, Extrapolation of Extreme and Fatigue Loads Using Probabilistic Methods, National Renewable Energy Laboratory, 2004.

[4] E. Castillo, Extreme Value Theory in Engineering, Academic Press, San Diego, Calif, USA, 1988.

[5] S. Kotz and S. Nadarajah, Extreme Value Distributions, Imperial College Press, London, UK, 2000.

[6] P. Ragan and L. Manuel, "Statistical extrapolation methods for estimating wind turbine extreme loads," Journal of Solar Energy Engineering, vol. 130, no. 3, Article ID 031011, 2008.

[7] Y. Gu, S. Li, H. Li, and Z. M. Guo, "A novel Bayesian extreme value distribution model of vehicle loads incorporating decorrelated tail fitting: theory and application to the Nanjing 3rd Yangtze River Bridge," Engineering Structures, vol. 59, pp. 386392, 2014

[8] S. Cabras and M. E. Castellanos, "An objective Bayesian approach for threshold estimation in the peaks over the threshold model," in Proceedings of the International Workshop on Objective Bayes Methodology, Padua, Italy, April 2009.

[9] C. Scarrott and A. MacDonald, "A review of extreme value threshold estimation and uncertainty quantification," RevstatStatistical Journal, vol. 10, no. 1, pp. 33-60, 2012.

[10] A. Tancredi, C. Anderson, and A. O'Hagan, "Accounting for threshold uncertainty in extreme value estimation," Extremes, vol. 9, no. 2, pp. 87-106, 2006.

[11] R. I. Harris, "Improvements to the 'method of independent storms," Journal of Wind Engineering and Industrial Aerodynamics, vol. 80, no. 1-2, pp. 1-30, 1999.

[12] R. L. Smith, "Extreme value theory based on the $r$ largest annual events," Journal of Hydrology, vol. 86, no. 1-2, pp. 27-43, 1986.

[13] Y. An and M. D. Pandey, "A comparison of methods of extreme wind speed estimation," Journal of Wind Engineering and Industrial Aerodynamics, vol. 93, no. 7, pp. 535-545, 2005.

[14] C. Mudersbach and J. Jensen, "Nonstationary extreme value analysis of annual maximum water levels for designing coastal structures on the German North Sea coastline," Journal of Flood Risk Management, vol. 3, no. 1, pp. 52-62, 2010.

[15] G. Villarini, J. A. Smith, F. Serinaldi, and A. A. Ntelekos, "Analyses of seasonal and annual maximum daily discharge records for central Europe," Journal of Hydrology, vol. 399, no. 3-4, pp. 299-312, 2011.

[16] C. Marty and J. Blanchet, "Long-term changes in annual maximum snow depth and snowfall in Switzerland based on extreme value statistics," Climatic Change, vol. 111, no. 3-4, pp. 705-721, 2012.

[17] D. Rivas, F. Caleyo, A. Valor, and J. M. Hallen, "Extreme value analysis applied to pitting corrosion experiments in low carbon steel: comparison of block maxima and peak over threshold approaches," Corrosion Science, vol. 50, no. 11, pp. 3193-3204, 2008.

[18] S. Bruni, M. Bocciolone, and S. Beretta, "Simulation of bridgeheavy road vehicle interaction and assessment of structure durability," International Journal of Vehicle Design, vol. 10, no. 1-2, pp. 70-85, 2003.

[19] J. X. Wang, N. X. Wang, Z. Y. Wang, Y. S. Zhang, and L. Liu, "Determination of the minimum sample size for the transmission load of a wheel loader based on multi-criteria decision-making technology," Journal of Terramechanics, vol. 49, no. 3-4, pp. 147-160, 2012.

[20] S. Sarata, N. Koyachi, T. Tubouchi, H. Osumi, M. Kurisu, and K. Sugawara, "Development of autonomous system for loading operation by wheel loader," in Proceedings of the 23rd International Symposium on Robotics and Automation in Construction (ISARC '06), pp. 466-471, Tokyo, Japan, October 2006.

[21] G. Ramazan, S. Faruk, and U. Abdurrahman, "Evim: a software package for extreme value analysis in matlab," Studies in Nonlinear Dynamics \& Econometrics, vol. 5, no. 3, pp. 213-239, 2001.

[22] P. A. Brodtkorb, P. Johannesson, G. Lindgren, I. Rychlik, J. Ryden, and E. Sjo, "WAFO-a Matlab toolbox for analysis of random waves and loads," in Proceedings of the 10th International Offshore and Polar Engineering Conference, vol. 3, no 4, pp. 343-350, Washington, DC, USA, 2000.

[23] J. R. M. Hosking, "Maximum-likelihood estimation of Parameter for the generalized extreme-value distribution," Applied Statistics, vol. 34, no. 3, pp. 301-310, 1985.

[24] R. B. D'Agostino and M. A. Stephen, Goodness-of-FitTechniques, Marcel Dekker, 1986.

[25] D. J. Shi, Practical Extreme Statistical Method, Tianjin Science and Technology Publishing House, Tianjin, China, 2006.

[26] J. H. Drew, A. G. Glen, and L. M. Leemis, "Computing the cumulative distribution function of the Kolmogorov-Smirnov statistic," Computational Statistics and Data Analysis, vol. 34, no. 1, pp. 1-15, 2000.

[27] H. Çalışkan, B. Kurşuncu, C. Kurbanoğlu, and Ş. Y. Güven, "Material selection for the tool holder working under hard milling conditions using different multi criteria decision making methods," Materials \& Design, vol. 45, pp. 473-479, 2013.

[28] J. Wang, Y. Liu, X. Zeng, Z. Zhou, N. Wang, and W. Shen, "Selection method for kernel function in nonparametric extrapolation based on multicriteria decision-making technology," Mathematical Problems in Engineering, vol. 2013, Article ID 391273, 11 pages, 2013.

[29] J.-J. Wang, Y.-Y. Jing, C.-F. Zhang, and J.-H. Zhao, "Review on multi-criteria decision analysis aid in sustainable energy decision-making," Renewable and Sustainable Energy Reviews, vol. 13, no. 9, pp. 2263-2278, 2009.

[30] X. L. Zhao and Q. J. Qi, “The establishment and application of fuzzy comprehensive model with weight based on entropy technology for air quality assessment," Procedia Engineering, vol. 7, pp. 217-222, 2010. 


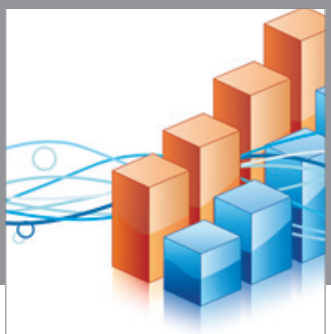

Advances in

Operations Research

vatem alat4

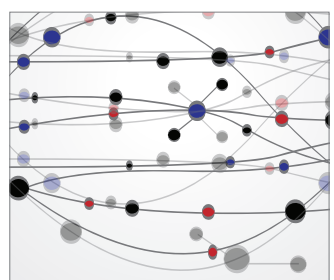

\section{The Scientific} World Journal
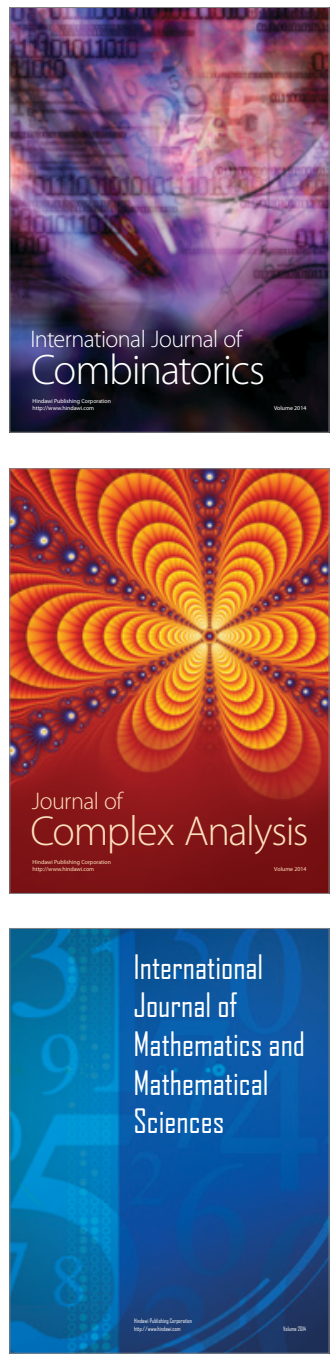
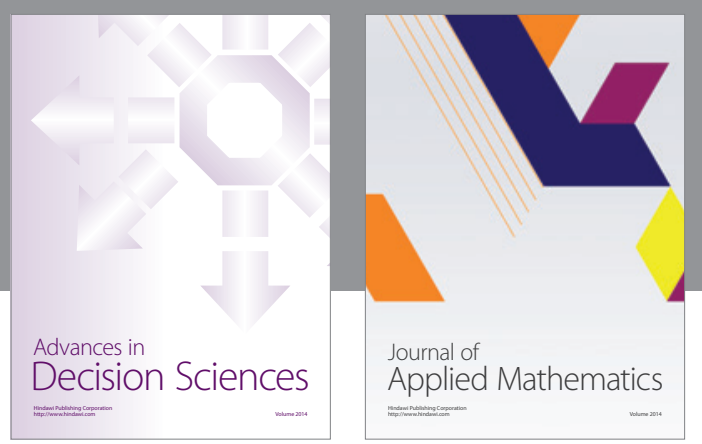

Algebra

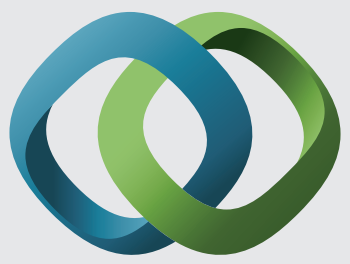

\section{Hindawi}

Submit your manuscripts at

http://www.hindawi.com
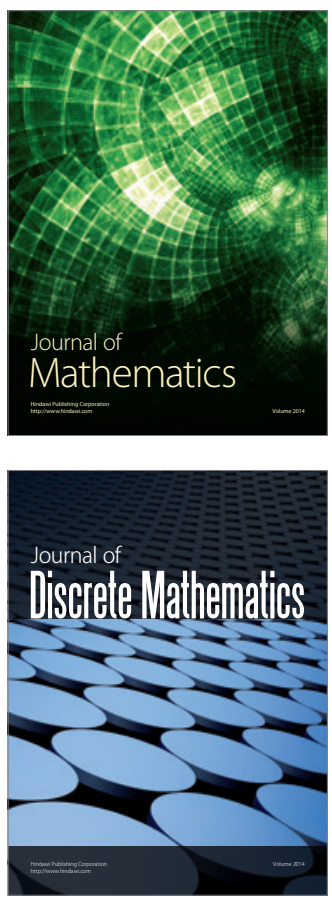

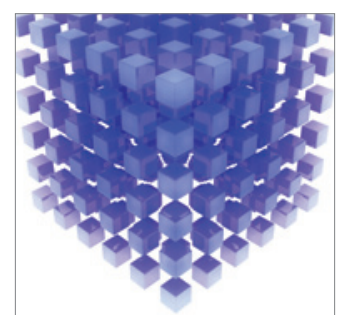

Mathematical Problems in Engineering
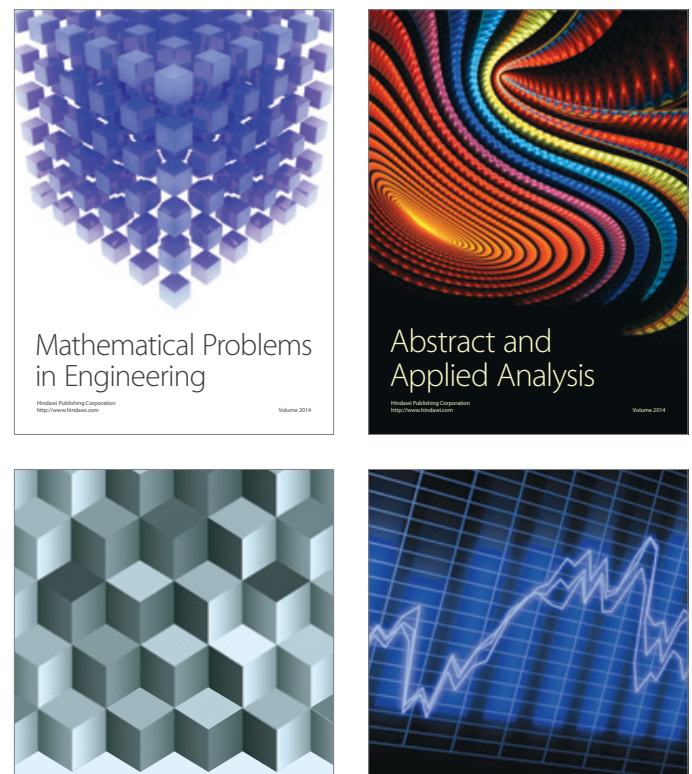

Journal of

Function Spaces

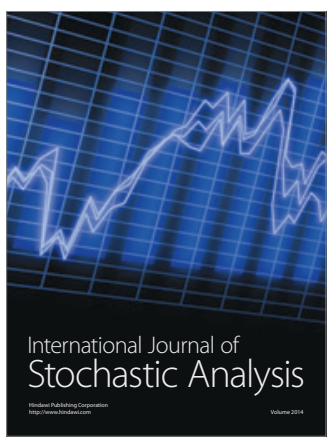

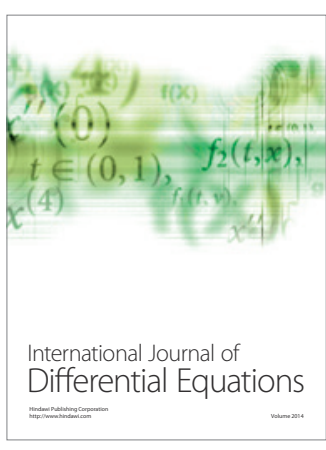
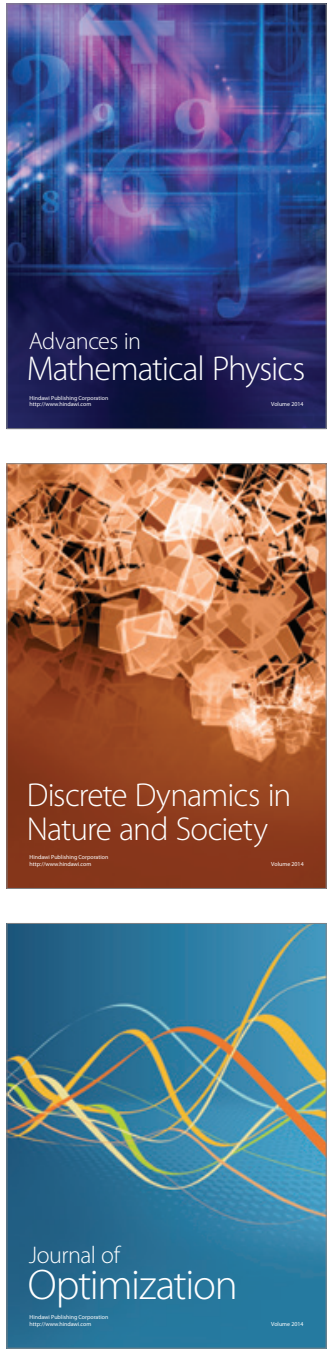